\title{
Comment coordonner laboratoires et essais en mer ? Détecteurs à ultrasons et formes d'innovation dans les marines françaises et anglaises
}

How to coordinate labs and open sea experiments? Forms of innovation in the French and English navies

\section{Benoit Lelong}

\section{OpenEdition}

\section{Journals}

Édition électronique

URL : http://journals.openedition.org/dht/1729

DOI : $10.4000 /$ dht. 1729

ISSN : $1775-4194$

Éditeur :

Centre d'histoire des techniques et de l'environnement du Cnam (CDHTE-Cnam), Société des élèves du CDHTE-Cnam

\section{Édition imprimée}

Date de publication : 1 décembre 2011

Pagination : 85-95

ISBN : 978-2-9530779-7-1

ISSN : 0417-8726

\section{Référence électronique}

Benoit Lelong, «Comment coordonner laboratoires et essais en mer ? Détecteurs à ultrasons et formes d'innovation dans les marines françaises et anglaises ». Documents pour l'histoire des techniques [En ligne], 20 | 2e semestre 2011, mis en ligne le 14 septembre 2012, consulté le 08 septembre 2020. URL : http://journals.openedition.org/dht/1729 ; DOI : https://doi.org/10.4000/dht. 1729 


\title{
Comment coordonner laboratoires et essais en mer? Détecteurs à ultrasons et formes d'innovation dans les marines françaises et anglaises*
}

\author{
Benoit Lelong \\ LATTS, Université de Paris-Est
}

\begin{abstract}
Résumé
L'article est consacré aux expérimentations réalisées en mer pour évaluer des technologies destinées aux navires militaires. Dans les années 1920, les marines nationales recoururent de plus en plus aux recherches de laboratoire pour améliorer leurs nouveaux équipements de détection et de communication. Les environnements matériels et humains accueillant les expériences se multiplièrent en conséquence, de l'évier à la cuve de test, du port à la haute mer. Des difficultés organisationnelles inédites se manifestèrent avec la nécessité nouvelle des coopérations entre chercheurs et ingénieurs, civils et militaires, scientifiques et marins. Elles furent redoublées par la rareté et le coût important des navires d'essai. Identités professionnelles, formes de normativité et réseaux sociaux s'agencèrent diversement selon les objets techniques. Toutefois, les collaborations et contrastes franco-britanniques mettent en évidence des différences assez stables, dépendant des institutions scientifiques et techniques nationales et des choix géostratégiques propres aux deux pays.
\end{abstract}

Résumés et mots clés en anglais sont regroupés en fin de volume, accompagnés des mots clés français

$\mathrm{D}$ 1925 à 1927, le physicien Paul Langevin et l'ingénieur Charles Florisson s'efforcent de mettre au point un phare U.S. (à ultrasons) pour le port de Calais. Au bout de ces trois années, le faisceau ultrasonore est jugé insuffisamment détectable par les navires, et le projet est finalement abandonné. Dans une lettre à Langevin, Florisson regrette la perte du site. "Calais est en effet pour nous une station d'expérience de premier ordre : nous ne possédons pas d'autre Station U.S. sur navire rapide où nous puissions travailler, sans trop de frais, durant des traversées courtes - et ceci très à portée de Paris $1 \%$. Cinq ans plus tôt, les deux hommes pouvaient facilement utiliser les infrastructures de test de la Marine à Toulon. Mais la relation avec les officiers de marine s'est dégradée: l'accès aux navires d'essai devient plus difficile, ce qui réduit les possibilités de validation des prototypes développés par les deux innovateurs'.

Ce cas illustre la fragilité des liens construits à l'époque entre chercheurs et marins participant à l'innovation industrielle et militaire. Depuis une dizaine d'années, l'historiographie a rendu particulièrement visible la variété contextuelle de ces collaborations en dépassant les travaux strictement monographiques. En travaillant sur le temps long menant de la première à la deuxième guerre mondiale, les historiens ont montré

1 Benoit Lelong, « Paul Langevin et la détection sous-marine, 1914-1929. Un physicien acteur de l'innovation industrielle et militaire ॥, Epistémologiques, vol. ॥, n 1-2, 2002, pp. 205-232. Lettre Florisson à Langevin, 18 février 1929, courrier SCAMSAGA, FL, carton 140.

\footnotetext{
* Pour l'essentiel, les archives utilisées sont issues du Fonds Langevin (École de physique et chimie, Paris) et du Service historique de la marine (Fort de Vincennes). Pour le cas anglais, quelques pièces proviennent des archives de l'Amirauté (The National Archives) et la plupart ont été communiquées par Sébastien Soubiran. Pour ces trois fonds, les abrévations utilisées en note sont respectivement FL, SHM et TNA.
} 
combien les coopérations entre expérimentateurs et militaires ont dépendu de multiples agencements de l'échelle des relations interpersonnelles jusqu'à celle des grands affrontements internationaux². On voudrait déployer ici une telle approche à propos des appareils ultrasonores - et ceci depuis la Grande Guerre jusqu'à la fin des années vingt. Confronter plusieurs technologies (le sondage vertical et la détection horizontale) et configurations nationales (les marines française et anglaise) visera à montrer le caractère éminemment local et négocié des formes de coordination. II s'agira ainsi d'apporter un modeste complément à des comparaisons internationales de bien plus grande ampleur, réalisées sur le radioguidage, le réglage du tir en mer, l'artillerie de terre et le repérage acoustique des batteries ${ }^{3}$.

\section{Des collaborations à l'épreuve des essais en mer}

Au cours de la décennie suivant l'armistice, les difficultés de coordination entre les chercheurs et les marins, et plus spécifiquement entre les expériences de laboratoire et les essais en mer, forment un motif récurrent dans les discours produits par les militaires. Ces déclarations résultent en partie de la réorganisation mise en œuvre au tout début des années vingt. Dès la fin de la guerre, la Marine entreprend en effet de poursuivre les collaborations mises en place pendant le conflit - l'exemplarité du travail mené sur les « appareils U.S. " (à ultrasons) lui donnant alors valeur de modèle. Par arrêté ministériel du 11 juillet 1921, ordre est donné de détacher la section de recherche scientifique du premier bureau de l'État-major Général, et de créer un État-major général de la recherche scientifique. Celvi-ci a dès lors pour fonction de diriger les quatre Centres d'Etudes de la Marine situés à Brest, Cherbourg, Rochefort et Toulon. Chacun de ces centres accueille une ou plusieurs Commissions d'Études Pratiques spécifiquement consacrées à un matériel particulier.

2 David Aubin, Patrice Bret dir., Le sabre et l'éprouvette. L'invention d'une science de guerre, 1914-1939, Paris, Éditions Noesis/Agnès Viénot, 2003 (14-18 Aujourd'hui. Today. Heute, n 6, pp. 39-172). Pour la période suivante, voir Amy Dahan, Dominique Pestre dir., Les sciences pour la guerre, 1940-1960, Paris, Éditions de l'EHESS, Paris, 2004.

3 Sébastien Soubiran, « De l'utilisation contingente des physiciens dans les systèmes d'innovation des marines française et britannique entre les deux guerres mondiales. Deux exemples: la conduite du tir des navires et la télémécanique ", thèse de doctorat d'histoire des sciences, université Paris 7, 2002 : Martina Schiavon, "Itinéraires de la précision. Géodésiens, artilleurs, savants et fabricants d'instruments en France, 1870-1930 ॥, thèse de doctorat d'histoire, EHESS, 2003.
Créé en mai 1920 à partir du Laboratoire de la guerre sous-marine, le centre de Toulon abrite alors neuf de ces commissions, dont celle de Détection sous-marine (CEP-DSM). Au sein de la Marine, les expérimentateurs de laboratoire ont désormais un statut précis (en termes de contrat de travail, de salaire, de gestion de carrière) et ils sont appelés " collaborateurs scientifiques ".

Cette complexification organisationnelle a rendu beaucoup plus saillants les décalages pratiques séparant les collectifs engagés dans l'innovation. En 1924, la Revue Maritime publie un article extrait d'un mémoire présenté par le lieutenant de vaisseau Alfred Baule à l'Association technique maritime et aéronautique. Après avoir présenté l'utilisation de la recherche scientifique par les marines de guerre anglaise, américaine, italienne et japonaise, le texte décrit l'organisation française et montre que deux populations ont bien du mal à travailler ensemble : d'une part les chercheurs et inventeurs, et d'autre part les marins, qu'il s'agisse des officiers dirigeant les navires de guerre ou des ingénieurs militaires en assurant les constructions et réparations dans les arsenaux. Entre le personnel des arsenaux et les inventeurs, affirme Baule, « il y a incompatibilité de tempérament. L'arsenal, soumis à une réglementation administrative très pesante, ne peut se plier aux retouches continuelles, aux recommencements que nécessitent les inventions, non plus qu'à l'extrême mobilité d'esprit qui caractérise généralement les chercheurs ". De telles difficultés de coordination sont particulièrement éprouvées par le laboratoire du Centre d'études de Toulon, dans l'organisation des essais en mer. Cet établissement, certes, est bien équipé pour l'expérimentation et le prototypage :

« Le Laboratoire se trouve donc bien outillé pour entreprendre la construction de modèles très divers et pour en effectuer les essais à terre. Mais pour mettre au point un appareil marin, quel que soit son but, il est indispensable de lui faire subir de nombreux essais à la mer. Là seulement, les travaux des Commissions et du Laboratoire peuvent trouver leur sanction définitive. Or, en ce qui concerne les moyens flottants d'expériences, il faut reconnaître que le Centre d'Études est bien mal outillé. II s'ensuit que le Centre d'Études se trouve obligé d'avoir recours à d'autres services (escadres, division d'instruction, flottille, etc.) pour essayer les appareils qu'il réalise. Procédé condamnable au premier chef, car rien n'est plus mauvais que de mettre un appareil non terminé à bord d'un bâtiment assurant un service 
normal ; les essais sans cesse interrompus par les appareillages obligatoires ne peuvent être suivis facilement par les inventeurs et les chercheurs; ils sont alors confiés en partie, sinon en totalité, au personnel de bord déjà surchargé par l'entretien de ses propres appareils. L'officier chef du service n'a bientôt plus qu'une seule idée, se débarrasser au plus vite de l'appareil en essai. En fait, si bien des inventions mettent dans notre marine des années à être mises au point, c'est uniquement parce qu'elles sont mises en essai sur des bâtiments qui ne sont pas faits pour cela, les inventeurs ne pouvant d'ailleurs suivre ces essais jusqu'au bout $)^{4}$.

Les tentatives de collaboration entre le laboratoire et le navire se sont heurtées aux différences de temporalités propres à ces deux espaces de pratiques. En mars 1927, le capitaine de vaisseau Hervé termine de rédiger un texte présentant «Le Centre d'Études de Toulon ॥ qui se termine par trois pages montrant que ses diverses composantes organisationnelles peinent à coopérer. D'une part, « les locaux affectés au Centre d'Études lors de sa création n'ont pas permis de réunir dansle même bâtiment le laboratoire etl'ensemble des commissions permanentes qui en dépendent; et dès lors la dispersion des divers organismes constitue une gêne pour l'étroite collaboration des collaborateurs et des commissions ॥. D'autre part, le personnel marin des moyens flottants du centre, les Eparges et l'Attentif, est insuffisant: «ces deux unités ne peuvent tenir ensemble la mer aussi fréquemment qu'il conviendrait ; les études de la CEP-DSM, par exemple, souffrent de cet état de choses ॥. Le développement des appareils U.S. est particulièrement pénalisé. De plus, le Centre d'études n'est pas autonome pour l'organisation des essais en mer : " il est peu d'études qu'il puisse pousser à bout sans le concours des services extérieurs et en tout premier lieu des directions de l'arsenal (les installations à bord, par exemple, impliquent toujours le concours des constructions navales). II faudrait, pour bien faire, que ce concours pût être accordé sans délai et que la direction en cause s'ingéniât à réduire au minimum tout retard qui dépend d'elle. Les exemples sont assez fréquents de la lenteur de certains travaux demandés : nous indiquerons seulement le cas des appareils U.S. des bâtiments et les transmissions des sous-marins. L'étude de ces questions a été singulièrement retardée par l'attente d'installations de bord du ressort des directions de l'arsenal. ॥ Enfin,

4 Alfred Baule, « La recherche scientifique dans les marines de guerre 1 , Revue Maritime, $2^{e}$ semestre 1924, pp. 480-500, pp. $490-491$ et 498. l'organisation des carrières militaires ne permet pas aux officiers de marine du service général (c'est-à-dire non spécialisés professionnellement comme le sont par exemple les médecins des services de santé) de suivre les objets techniques des premières expérimentations jusqu'aux derniers essais en mer. "Les officiers du service général, en effet, ne font pas toujours un séjour assez prolongé pour voir aboutir les études qui leur sont confiées ; parfois même il arrive, et la chose est fâcheuse, qu'aucun des officiers du service général ayant participé à la mise sur pied d'un projet ne puisse voir les essais du prototype ». Cette instabilité est préjudiciable car ces officiers représentent le seul personnel de Toulon familiarisé avec les usages en mer des équipements en situation ordinaire. II faut donc que de tels profils soient incités à séjourner longuement au Centre ; "là est leur place logique et légitime puisqu'ils y peuvent collaborer avec fruit à cette œuvre primordiale : la préparation du matériel de combat dont ils ont déjà étudié la mise en œuvre et l'utilisation ». De telles déclarations, fréquentes chez les officiers, sont également repérables chez les ingénieurs et les chercheurs au sein de la Marine. Au final, c'est un discours de rareté que tiennent l'ensemble des acteurs vis-à-vis des moyens matériels et humains nécessaires pour les essais en mer. Et les vives tensions entre les divers services renvoient pour une part à des stratégies de contrôle de ces ressources, des navires destinés aux essais comme du personnel marin techniquement capable de les mettre en œuvre 5 .

Au cours des années vingt, les militaires jugent de plus en plus sévèrement tous ces décalages, leur imputant des conséquences néfastes pourl'intérêt des expérimentations menées par les chercheurs recrutés par la Marine. En 1927, le capitaine de frégate Dubois, commandant le laboratoire de Toulon, critique une étude électronique des projecteurs U.S. menée par Gaston Laville, un des collaborateurs scientifiques, affirmant qu'il s' agit de « mesures de laboratoire faites dans des conditions qui ne sont pas celles d'emploi à la mer $11^{6}$. Les écarts entre les essais à terre et en mer sont jugés d'autant plus problématiques que la légitimité des recherches expérimentales demeure fragile pendant cette période. Plus globalement, c'est l'ensemble du laboratoire toulonnais qui se voit ainsi contesté. En 1931, le travail mené à Toulon est critiqué par le capitaine de frégate Raymond Fénard commandant l'État-major général de la recherche

5 Capitaine de vaisseau Hervé, "Le Centre d'Études de Toulon », juillet 1927, SHM, 1BB8, carton 140.

6 Gaston Laville, « Essais par voie électrique des projecteurs U.S. ॥, 9 novembre 1927, suivi de « Observations du capitaine de frégate directeur $\gg, \mathrm{FL}, \mathrm{L} 15 / 20$.. 
scientifique. « La progression de ces études dépend beaucoup plus de la nature du problème scientifique que de l'utilité militaire de l'appareil recherché. II est donc tout à fait possible que le [Centre de Toulon], plaçant toutes ces études sur le même plan, propose prochainement des appareils dont l'intérêt militaire ne sera que secondaire ou peut-être nul $17^{7}$. E† de fait, une réforme d'ensemble est menée en 1931 qui accroît encore I'hétéronomie du laboratoire vis-à-vis des Commissions d'études pratiques. Cette transformation prend notamment acte de la rareté des ressources disponibles pour les essais en mer : plus qu'auparavant, elles seront réservées aux études dont l'intérêt militaire apparaît le plus évident.

\section{La mobilisation scientifique, 1915-1918}

En pointant les difficiles coordinations entre expérimentation de laboratoire et essais en mer, les acteurs des années vingt incriminèrent souvent l'organisation mise en place dans l'immédiat aprèsguerre. Le fait est que la situation était fort différente à la fin du conflit, qui marqua plus généralement une véritable rupture dans les politiques publiques de l'innovation'. Plus exactement, c'est le moment suivant la mobilisation scientifique de 1915 qui présente des traits spécifiques dans la contribution des laboratoires à l'étude des équipements et au renseignement?. On sait que cette remobilisation ne résulta pas, de la part des militaires, d'une décision spontanée de faire appel aux scientifiques ${ }^{10}$. II s'est plutôt agi d'un besoin politiquement créé par la communautésavante àla faveurdescirconstances, et en particulier de l'évidente supériorité technologique de l'ennemi pour certains équipements. La Direction des Inventions intéressant la défense nationale est créée en novembre 1915 et confiée à Paul Painlevé qui organise dès lors la mobilisation scientifique. Sous l'autorité du Ministère de l'Armement à partir de décembre 1916, mais indépendante de la Marine, la Direction entreprend notamment de développer la détection acoustique aérienne et sous-marine ${ }^{11}$. Dès

7 Note du commandant de l'EMGRS destinée à l'EMG, 19 octobre 1931, SHM, 1BB2, carton 237.

8 Gabriel Galvez-Behar, La République des inventeurs. Propriété et organisation de l'innovation en France, 1791-1922, Rennes, Presses Universitaires de Rennes, 2008.

9 Patrice Bret, "Les laboratoires français et l'étude des munitions et matériels allemands pendant la Grande Guerre ॥, Cahiers du CEHD, n³3, 2008, pp. 7-32.

10 Anne Rasmussen, « Mobiliser, remobiliser, démobiliser. Les formes d'investissement scientifique en France dans la Grande Guerre 11, 14/18 Aujourd'hui. Today. Heute, 6, 2003, pp. 49-60.

11 Yves Roussel, «L'histoire d'une politique des inventions, le début de la guerre, le U-Boot allemand apparut comme une arme devant laquelle la flotte française était impuissante. Signe de son inquiétude, la Marine publia un Bulletin de la Guerre Sous-Marine recensant mensuellement les navires torpillés et coulés. Ses services techniques ne s'avérant guère capables de trouver une parade jugée efficace, les physiciens furent sollicités. Trois centres furent établis pour mettre au point des dispositifs de repérage des sous-marins à Toulon surtout, mais aussi à Brest et Cherbourg ${ }^{12}$.

Fin 1914, un ingénieur russe, Constantin Chilowski, suggère d'utiliser les ultrasons pour la détection des sous-marins. Son idée est d'utiliser pour cela des postes émetteurs TSF, car leurs fréquences sont proches de celles des ultrasons. Painlevé est intéressé et, en 1915, il demande à Langevin de collaborer avec Chilowski. En parallèle, l'un et l'autre contribuent également à des travaux de balistique sur l'optimisation des obus et des tuyères. ${ }^{13}$ Les deux hommes se mettent au travail à l'École de physique et de chimie avec deux anciens de cette école, Marcel Tournier et Fernand Holweck. En mars, un premier émetteur est au point, constitué d'un condensateur à lame de mica. Le récepteur est un microphone à charbon. L'ensemble est testé dans la Seine, et donne satisfaction. Dès le mois de mai 1916, un brevet est déposé au nom de Langevin et Chilowski. À la fin de l'année, Langevin décide d'utiliser la piézoélectricité pour détecter les ultrasons. Un premier récepteur à quartz est construit en mars et testé en avril à Toulon en remplacement du microphone. Encouragé par ce succès, Langevin décide d'utiliser la piézoélectricité également pour émettre les ultrasons. En avril 1917, l'émetteur à quartz fonctionne, mais les signaux émis sont trop faibles. Langevin décide de les amplifier en utilisant la résonance, en faisant vibrer la lame de quartz selon sa fréquence propre. Comme cette fréquence propre est trop grande, et produit des ultrasons très rapidement absorbés, Langevin la modifie en insérant la lame de quartz entre deux lames d'acier. L'appareil

1887-1918॥, Cahiers pour l'histoire du CNRS, 3, 1989, pp. 19-57 ; Gabriel Galvez-Behar, "Le savant, l'inventeur et le politique. Le rôle du sous-secrétariat d'état aux inventions durant la première guerre mondiale", Vingtième siècle. Revue d'histoire, 85 , 2005/1, pp. 103-117.

12 Ernest Esclangon, «Sur la détection sous-marine et les questions scientifiques qui s'y rattachent ", Rapport secret au SousSecrétariat de la Marine de Guerre, 28 juillet 1918, FL, L15/01. 13 Claudine Fontanon, "L'obus Chilowski et la soufflerie balistique de Paul Langevin: Une recherche militaire oubliée de la mobilisation scientifique (1915-1919) ॥, dans Dominique Pestre dir., Deux siècles d'histoire de l'armement. De Gribauval à la force de frappe, 2005, CNRS éditions, pp. 81-108. 
ainsi constitué, un triplet quartz-acier, fonctionne à la fois comme émetteur et comme récepteur : les deux fonctionnalités sont maintenant réunies dans un seul et même artefact. Les nouveaux appareils sont construits fin 1917, testés dans la Seine en février 1918, et à Toulon en avril 1918. En parallèle, la Marine a établi à Toulon un Laboratoire de la guerre sousmarine, dirigée par le capitaine de vaisseau Émile Moysan, avec une cuve pour tester l'appareil sur place et un navire pour les essais en mer. En juin 1918, le dispositif est considéré comme définitivement au point, et présenté comme tel dans plusieurs rapports adressés à la Marine ${ }^{14}$.

Par contraste avec les temps de paix, ces travaux de guerre témoignent d'une relative fluidité dans la circulation et l'accès aux divers lieux de mise à l'épreuve des technologies - un contraste qui n'a rien pour surprendre les historiens des techniques. La sécurité nationale implique néanmoins de nombreuses résistances à négocier, ne serait-ce que les multiples documents officiels à obtenir et faire signer pour autoriser les déplacements (des pièces que l'on retrouve aujourd'hui dans des archives comme celles de Langevin). À ce titre, un important rôle de médiation fut joué par Maurice de Broglie, basé à Toulon et rattaché à la Direction des inventions : diplômé de l'École navale et officier de marine de 1897 à 1908, il se consacrait depuis à la physique expérimentale, disposant de son propre laboratoire et faisant partie du groupe de recherche de Langevin. Painlevé et lui participaient tant au monde de la défense que de la recherche ${ }^{15}$. Avec leur soutien, la détection ultrasonore put se développer selon un modèle séquentiel, les objets techniques étant d'abord testés au laboratoire, puis dans la Seine, puis à Toulon en bord de mer, et enfin au large à bord des navires.

En Angleterre, les premières attaques des sousmarins allemands déclenchent immédiatement une très vive inquiétude. Dès septembre 1914, soit un mois après le commencement des hostilités, la Navy offre des récompenses pour leur repérage ou leur capture, et sollicite l'ensemble de la population, se déclarant intéressée par toute suggestion d'un procédé de détection sous-marine. Les scientifiques exploitent ce mouvement, déploient un actif lobbying pour déclencher une mobilisation scientifique, ce qui

14 Benoit Lelong, "Paul Langevin et la détection sousmarine 11, op. cit.

15 Claudine Fontanon, Robert Frank dir., Paul Painlevé (1863-1933). Un savant en politique, Rennes, Presses Universitaires de Rennes, 2006: Mary-Jo Nye, « Aristocratic Culture and the Pursuit of Science. The De Broglies in Modem France II, Isis, 88, 199, pp. 397-421. rencontre un accueil favorable chez A. J. Balfour, le First Sea Lord. Sous son impulsion est créé le BIR (Bureau of Invention and Research), un organe indépendant de la Navy et principalement composé de scientifiques. Ernest Rutherford y joue un rôle central, stimule les travaux sur la détection sousmarine, impliquant notamment Albert Beaumont Wood, un jeune expérimentateur qu'il a formé à la physique fondamentale dans son laboratoire de l'université de Manchester. Antérieurement avait été créée à Hawkcraig une station expérimentale de détection sous-marine dirigée par le capitaine C. P. Ryan. L'arrivée des scientifiques du BIR conduit immédiatement à des conflits avec les officiers de marine. Wood se plaint auprès de Rutherford du refus de Ryan de leur accorder un accès autonome à ses sous-marins et navires d'essai. Les physiciens quittent finalement Hawkcraig pour la nouvelle station du BIR installée à Harwich, également en bord de mer. De nombreux autres centres apparaissent, au nombre d'une trentaine, dépendant soit de la Navy, soit du BIR. Les travaux sur la détection des sous-marins sont menés par de multiples groupes exploitant des expertises locales, d'où un grand nombre de procédés différents. Comme en France, les travaux se concentrent d'abord sur des dispositifs d'écoute passive, principalement ici sur les hydrophones ${ }^{16}$.

En août 1915, Painlevé suggère une coopération scientifique entre la France et l'Angleterre, mais la Navy décline afin de protéger la confidentialité de ses travaux et les brevets de ses inventeurs. Une visite est néanmoins organisée : deux physiciens anglais viennent s'informer à Toulon, la Marine refusant toutefois qu'on leur montre les appareils ultrasonores. Les scientifiques du BIR réclament alors une collaboration avec Langevin. La Navy refuse, mais doit céder quand l'ordre est donné par Balfour en octobre. En mars 1917, Langevin donne une conférence à Londres sur les ultrasons. Le Bureau of Invention and Research lance deux groupes de scientifiques travaillant sur les technologies françaises (à Finsbury sur les lampes électroniques et à Harwich sur la piézoélectricité du quartz). En mai, Rutherford passe par Toulon avant de participer à la première conférence interalliée sur la détection sous-marine à Washington en juin 1917 (où il décrit le triplet quartzacier). Une deuxième conférence a lieu à Paris en octobre 1918, marquée par des débats sur la priorité et les droits respectifs des inventeurs anglais et français en matière de brevet. Un point notable de

16 Willem Hackmann, "Underwater acoustics and the Royal Navy, 1893-1930 II, Annals of science, 36, 1979, pp. 255-278. 
cette coopération internationale est que les Anglais ne sont pas parvenus à importer du quartz du Brésil, de Madagascar et du Cachemire, et qu'ils ont donc durablement dépendu de Langevin pour la fourniture des quartzs et pour leur certification. Celui-ci, en effet, s'investit personnellement dans cette affaire, trouve d'abord un cristal de $25 \mathrm{~cm}$ chez l'opticien parisien Ivan Werlein, puis obtient 4 tonnes de Bornéo et Madagascar. Les dix premiers détecteurs à ultrasons de la Royal Navy seront construits grâce à des lames provenant d'un stock trouvé à Bordeaux et fournies par la France ${ }^{17}$.

Ces liens et contrastes franco-britanniques invitent à comparer les configurations nationales. Dans les deux cas, la différence est nette avant et après la mobilisation scientifique : les physiciens jouèrent un rôle décisif dans le développement des premiers détecteurs à ultrasons. D'importantes contraintes de confidentialité, imposées par les militaires, purent être finalement levées. Eł dans un cas comme dans l'autre, l'accès aux navires pour la conduite des tests représentait un enjeu critique. Un trait frappant est que les Français, malgré une évidente infériorité de moyens, organisèrent une coopération entre expérimentateurs et marins qui conduisit à une solution technique, le triplet quartz-acier, finalement transférée et incorporée par les Anglais dans leurs propres dispositifs de détection.

\section{L'après-guerre en France : les sondeurs}

Après la fin du conflit, les formes de coordination entre le laboratoire et la mer vont considérablement évolver. Ces transformations tiennent en partie aux technologies nouvelles investies par les innovateurs. À Toulon, Langevin a rencontré Pierre Marti, du service d'hydrographie de la Marine, et Florisson, un ingénieur du Centre d'études. Ensemble, ils décident d'améliorer la technique du sondage, centrale pour les relevés hydrographiques. Leurs travaux commencent en 1919 et mènent à l'installation en février 1922 d'un prototype sur l'aviso Ville d'Ys, qui part pour une mission d'hydrographie entre Terre Neuve et l'Islande. Le sondeur ultrasonore est finalement breveté par Langevin et Florisson en décembre 1923 et mis en service par la Marine en mai $1924^{18}$.

Le contraste est d'importance avec les détecteurs de sous-marins, qui étaient en 1915 des objets techniques sans précédents. Développer le sondage

17 Willem Hackmann, Seek and strike. Sonar, anti-submarine warfare and the Royal Navy, 1914-1954, Londres, HMSO, 1984, pp. 80, 85-89.

18 Charles Florisson, « Sondage ultrasonore. Historique des essais de sondage à Toulon », 18 novembre 1924, FL, L136/02. acoustique - c'est-à-dire utilisant le son et non le plomb et le fil d'acier - n'était en 1919 ni une idée neuve, ni une initiative spécifiquement française. Plusieurs brevets de sondeurs basés sur l'écho obtenu par des explosions sous-marines avaient été déposés dans les années 1880 en Angleterre, en Allemagne et en France. Depuis le XIXe siècle, dans la plupart des grandes nations industrialisées, déterminer la profondeur du fond était essentiel pour plusieurs activités - notamment l'établissement des cartes marines par les services d'hydrographie militaire et la pose de câbles sous-marins par les compagnies de télégraphie ${ }^{19}$. En 1912, le naufrage du Titanic par un iceberg stimula les études sur la détection des corps immergés et provoqua une nouvelle série de brevets - la même année par le météorologiste anglais Lewis Fry Richardson et par le physicien allemand Alexander Behm, et en 1914 par l'ingénieur américain Reginald Aubrey Fessenden. Tous ces dispositifs ne furent que des initiatives locales sans réel prolongement industriel et commercial. Après 1918, l'expertise en détection sous-marine constituée pendant la guerre fut exploitée par la plupart des marines nationales pour développer le sondage. Aux États-Unis, des prototypes sont construits par le groupe de scientifiques dirigé par Harvey C. Hayes à la Naval Engineering Experimental Station de Annapolis. En Allemagne, Behm crée en 1920 à Kiel une société pour produire et commercialiser son invention, la Behm Echolot Gesellschaft20.

En France, le développement des sondeurs U.S. repose sur une configuration assez proche de celle déployée pendant le conflit, centrée sur l'émetteur-récepteur d'ultrasons, procédant par des tests successifs s'éloignant progressivement de l'évier du laboratoire jusqu'au navire en pleine mer. Deux personnes, Langevin et Moysan, ont une autorité incontestable sur les opérations et s'efforcent à l'évidence de reproduire les manières de faire et d'interagir qu'ils avaient stabilisées à la fin de la guerre. Mais cette organisation de l'innovation va évolver à la suite de la réorganisation décidée en 1921, et connaître de notables infléchissements selon la technologie concernée. Chaque configuration sociotechnique va dépendre d'agencements hautement idiosyncrasiques entre les acteurs en présence. II va s'agir notamment des Commissions d'études pratiques,

19 Helen M. Rozwadowski, « Technology and ocean-scape : defining the deep sea in mid-nineteenth century "1, History and technology, vol.17, n³, 2001, pp. 217-247.

20 Sabine Höhler, " Depth records and ocean volumes : ocean profiling by sounding technology, 1850-1930 ॥, History and technology, vol.18, n², 2002, pp. 119-154, pp. 129-132. 
celle d'artillerie de côte, celle d'optique et télémétrie, et celle de mines et grenades. En 1922, le centre intègre les trois Commissions créées depuis, celle de Détection sous-marine (CEP-DSM), celle de télégraphie sans fil, et celle des sous-marins. L'éventail des technologies développées à Toulon connaît ainsi une très forte diversification ${ }^{21}$.

Les Commissions d'études pratiques, uniquement composées de militaires, sont des organismes spécialisés chargés de mettre au point des équipements nouveaux. Elles servent d'interface entre l'industrie et les " utilisateurs" (ou plutôt les services de la Marine qui s'en font les porte-paroles, c'est-ò-dire l'État-major général, le Service hydrographique et les Directions d'artillerie navale et des constructions navales) établissant les cahiers des charges et entretenant des relations suivies avec les constructeurs. Une référence souvent citée est le premier télémètre stéréoscopique français, mis au point par la Commission d'optique et de télémétrie, et produit ensuite par la Société d'optique et de mécanique de haute précision - une réalisation perçue comme exemplaire parce qu'elle permit la mesure rapide de la distance d'un périscope de sous-marin, et parce qu'elle affranchit la Marine de son précédent fournisseur, l'allemand Carl Zeiss. Durant les années 1920, le laboratoire de Toulon apporte progressivement une triple assistance aux Commissions. Tout d'abord, son personnel scientifique se charge des recherches les plus fondamentales lou trop coûteuses ou trop confidentielles pour être confiées à l'industrie). Le laboratoire de Toulon assure également la construction des prototypes - disposant à cette fin d'un bureau de dessin, de cinq ateliers et d'une trentaine d'ouvriers. II devient enfin un service de veille scientifique et technique : en 1927, sa bibliothèque est abonnée à près de quatre-vingts revues, comprend un millier de volumes et édite un bulletin bibliographique hebdomadaire ; c'est à ce titre que sont entretenus des liens avec des physiciens, les « collaborateurs extérieurs " - parmi lesquels Paul Langevin, Jean Perrin, Jean-Jacques Trillat, Marcel Tournier, Fernand Holweck, Léon Brillouin et Jean Becquerel22.

Un point essentiel est que les Commissions d'Études Pratiques obtinrent graduellement de nouveaux équipements et personnels facilitant une prise d'autonomie vis-à-vis du laboratoire de Toulon. Sous le commandement du capitaine de frégate Héritier, la CEP-DSM devint un pôle d'expertise en matière de détection ultrasonore, et se constitua en point de

21 Capitaine de corvette Faurie, "Organisation du Centre d'Études de Toulon ॥, 24 novembre 1922, SHM, 1BB8, carton 136. 22 Capitaine de vaisseau Hervé, "Le Centre d'Études de Toulon », op. cit. passage obligé à l'intérieur du système. En octobre 1923, Héritier s'oppose à une dépêche ministérielle lui imposant de dispenser une formation à l'École de TSF, arguant que celle-ci n'a « ni le matériel nécessaire à cette instruction, ni le bâtiment indispensable pour la formation du personnel, ni le personnel instructeur » et réclame que la formation soit délivrée à la Commission ${ }^{23}$. Ultérieurement, la commission s'impliqua aussi dans la certification des appareils. Ses actions concoururent, à des degrés divers, à la constitution et à la préservation d'un monopole dans le développement de la technologie ultrasonore et dans la formation de ses utilisateurs.

Un trait notable des actions entreprises par la CEPDSM est la réhabilitation des fréquences audibles en sus des fréquences ultrasonores dans la détection sous-marine, ce que traduit la généralisation du terme "écoute " pour désigner des matériels et des formations. Les premiers résultats produits sont l'occasion de remarques acerbes dirigées contre les expérimentations de laboratoire. En février 1923, la commission donne les conclusions d'une importante campagne de tests en mer réalisés sur les quatre principaux dispositifs d'écoute (IPM, tube C, appareil K, Walser). Au laboratoire, le Walser est celui dont la portée est la plus réduite. Mais tous les autres instruments s'avèrent beaucoup moins performants en situation ordinaire d'usage : trop sensibles, ils exigent une grande immobilité du navire et de son personnel. Leur portée pratique est donc inférieure à celle du Walser « car toutes les conditions précédentes ne peuvent jamais être réalisées sur un bâtiment de guerre en navigation courante, même avec une grande discipline du silence à bord $11^{24}$. Les essais réalisés en laboratoire étaient critiqués en contestant l'extension de leurs résultats aux situations d'usages en mer.

Les relations entre la CEP-DSM et le laboratoire peuvent être saisies à partir des deux principales classes d'interface impliquées dans la détection sousmarine. La première est l'écoute directe par caisson et stéthoscope : les activités sont exclusivement du ressort de la commission, sans participation du laboratoire, et témoignent d'une logique de progression très incrémentale. La deuxième est représentée par la transmission électrique etles microphones. La Commission confie au laboratoire le développement d'appareils assez récents, les microphones électromagnétiques. Les actions mises en œuvre montrent que c'est elle, et non

23 Héritier, "Réponse à la DM 1761 EMG4 en date du 22 septembre 1923 ॥, 20 octobre 1923, SHM, 1BB8, 63.

24 CEP-DSM, «P.V. de la séance du 23 février 1923 au sujet de la valeur comparative des divers appareils d'écoute réglementaires et de leurs conditions d'emploi ॥, SHM, 1BB8, carton 63. 
le laboratoire, qui assure le commandement et effectue la quasi-totalité du développement. Le laboratoire n'est impliqué que pour les tâches requérant une expertise et des instruments que la CEP-DSM ne possède pas. La même logique prévaut pour les savants extérieurs. Un appareil très nouveau, un prototype de microphone à quartz, a été proposé par Langevin : il n'éveille aucun intérêt de la part de la Commission et les études sont arrêtées jusqu'à ce que le physicien intervienne personnellement à Toulon. Au total, le laboratoire n'est guère impliqué dans les prises de décision : la commission s'efforce de lui assigner un rôle de simple exécutant, à qui on confie des tâches circonscrites, qui n'engagent pas la totalité des objets techniques ${ }^{25}$

Repérable pour la détection acoustique ou ultrasonore des sous-marins, cette configuration ne se retrouve en revanche pas pour le sondage. En 1924, les sondeurs sont devenus une classe de sonars spécifique, et le rapport trimestriel de la CEP-DSM change de structure pour rendre compte de cette différenciation. D'autres appareils viennent bientôt renforcer cette spécificité et en cliver les usages : l'analyseur Florisson pour la navigation courante (la profondeur est indiquée par un spot lumineux frappant une échelle graduée à chaque retour d'écho) et l'enregistreur Marti pour l'hydrographie (avec tracé d'une courbe en continu). À partir de 1924, la Commission classe les bâtiments de la Marine en trois catégories (A pour la navigation courante, B pour l'hydrographie occasionnelle, C pour l'hydrographie permanente), chacune de ces trois catégories recevant des appareils et des notices d'utilisation spécifiques (analyseur pour les A, analyseur et enregistreur pour les $B$, enregistreur en double exemplaire pour les $\mathrm{C}$ ). Un domaine socio-technique existait déjà autour du sondage, dû à la pré-existence de sondeurs acoustiques et à la présence des ingénieurs hydrographes ${ }^{26}$.

La spécificité des sondeurs ne concerne pas uniquement la forme matérielle des artefacts, leurs usages, leurs destinataires et leurs modes d'emploi. Elle renvoie également à des réseaux et à des formes particulières de coopération entre physiciens et militaires, ce qu'illustre le développement des sondeurs pour grandes profondeurs. En 1923, de retour d'une mission d'hydrographie en Algérie, Marti signale à Langevin que les sondeurs ultrasonores sont inopérants aux grandes profondeurs, et qu'il a dû recourir au sondage acoustique par détonation au-delà de 200 mètres. Dans son laboratoire parisien, Langevin poursuit l'étude de

25 Rapports trimestriels du Laboratoire du Centre d'études de Toulon, SHM, 1BB8, cartons 136-137-138.

26 Courrier Héritier à Moysan, 12 décembre 1924 ; Moysan au préfet maritime de Toulon, 16 décembre 1924, SHM, 1BB8, carton 136. la puissance d'émission des ultrasons. En mars 1924, un nouveau sondeur est opérationnel, capable de sonder entre 200 et plusieurs milliers de mètres. Le commandant du centre de Toulon demande alors au préfet maritime que l'appareil soit confié au Service hydrographique d'Algérie moyennant une opération comptable ${ }^{27}$.

Le développement de ces nouveaux sondeurs dessina donc le même réseau et les mêmes conventions de coopération que les précédents. Au centre du processus se trouvaient les hydrographes, les physiciens et les ingénieurs de la SCAM (l'entreprise fabriquant les appareils U.S.). Les officiers de marine étaient régulièrement informés et signaient les autorisations officielles, mais ne prenaient guère part aux décisions. En revanche, un rôle essentiel était joué par Langevin, qui mobilisait toute son expertise de la piézoélectricité pour repenser les détails du triplet quartz-acier. Comme pour les sondeurs précédents, la constitution et la certification de l'artefact obéissait ensuite à une logique plutôt séquentielle, traversant successivement l'évier du laboratoire de Langevin à Paris, la cuve du Centre d'études de Toulon, et la Méditerranée pour les essais en mer. Le contraste avec les techniques d'écoute sousmarine est particulièrement net. II illustre la diversification des formes d'innovation faisant suite à la réorganisation de 1921. Les modalités de coordination adoptées deviennent alors très variables selon la technologie développée et la configuration d'acteurs en présence.

\section{Contraste anglais : les asdics}

Comme en France, une alliance entre physiciens, militaires et ingénieurs se met en place en Angleterre autour de la détection sous-marine, une configuration présentant plusieurs caractères propres, d'ordre organisationnel et symbolique, qui permettent de saisir en retour les spécificités du cas français ${ }^{28}$. En 1918, afin de résoudre les conflits entre scientifiques et militaires (comme ceux survenus à Hawkcraig), le BIR est dissous et remplacé par un unique organisme de la Navy, le Department of Experiment and Research. L'année suivante, tous les scientifiques restant à disposition sont transférés à la station de Shandon, en attendant de rejoindre le grand laboratoire de recherche en projet dès la fin du conflit et finalement créé en 1921. Plus tard, certains sont incorporés dans trois autres centres d'expertise et de formation, la AntiSubmarine School (pour y travailler sur les procédures

27 «Sondage aux grandes profondeurs par ultra-sons », courrier de Moysan au préfet maritime de Toulon, 15 janvier 1924, SHM, 1BB8, carton 136.

28 Sur la comparaison entre les formes d'innovation dans les marines françaises et anglaises, la référence principale est Soubiran, «De l'utilisation contingente des physicien », op. cit. 
de détection), la Signal School (sur les émetteursrécepteurs d'ondes ultrasonores et radio), et la Mining School (sur les hydrophones). Ainsi, comme pendant la guerre, une particularité anglaise est le grand nombre de sites accueillant des civils formés à la recherche expérimentale ${ }^{29}$.

Un premier élément de contraste avec la France est en effet la puissance institutionnelle, matérielle et financière de la Royal Navy (les redevances à payer pour les brevets français sont calculées sur la base d'un rapport de un à quatre entre les deux flottes). La Navy bénéficie des certitudes ancrées dans l'espace public et la classe politique - à savoir que l'Angleterre est la première nation maritime du monde, que le contrôle des mers est de première importance pour sa sécurité et celle de son empire colonial, et que la flotte est un instrument essentiel de sa politique internationale. Dès 1918, l'Admiralty est convaincue que cette hégémonie est directement menacée par les nouvelles technologies de la guerre sous-marine, et que sa préservation passera par l'intégration de la recherche académique - et elle mène campagne avec le soutien de la communauté savante pour obtenir un grand laboratoire de recherche. L'Admiralty Research Laboratory créé en 1921 est installé à Teddington, une petite ville de la banlieve londonnienne abritant également le National Physical Laboratory, ce qui garantit la proximité avec la vie scientifique et technique de la capitale. Trente-six civils, scientifiques ou ingénieurs de formation, y travaillent en 1927. Certains sont des physiciens titulaires d'un doctorat (tels A. B. Wood, F. B. Young ou R. T. Beatty) qui s'efforcent une fois recrutés de publier dans les revues savantes. L'itinéraire de Wood est particulièrement significatif : il entre à l'ARL en 1921 et y prend la direction du groupe d'acoustique en $1927^{30}$. Au laboratoire de Toulon, certains " collaborateurs scientifiques " ont un doctorat, d'autres sont issus de l'EPC qui est très loin d'être une école de second rang, mais ils ne furent jamais plus de 5 ou 6 et n'atteignirent jamais un effectif jugé suffisant pour distinguer plusieurs groupes. Même si l'organisation du laboratoire de Toulon prit modèle comme l'ARL sur le National Physical Laboratory, certains profils furent probablement perdus pour le recrutement du fait de la localisation à Toulon, donc loin de Paris - et ce malgré la proximité de Marseille, alors un des grands centres provinciaux d'excellence scientifique et technique ${ }^{31}$.

29 Willem Hackmann, "Sonar, wireless telegraphy and the Royal Navy: scientific development in a military context, 1890-1939 1), dans Nicolaas Rupke dir., Science, politics and the public good, Londres, MacMillan, 1988, pp. 90-118.

30 Notice biographique du fonds Albert Beaumont Wood, TNA, archives de l'Admiralty Research Laboratory, série ADM 218.

31 Lelong, «Paul Langevin et la détection sous-marine », op. cit.
Un deuxième trait particulier du dispositif anglais est l'extrême importance stratégique de la détection des sous-marins. En 1921, à la conférence de Washington qui conduira au traité de Versailles, les Anglais essaient d'obtenir l'abolition du sous-marin comme arme de guerre. Comme les Français et les Américains s'y opposent, Balfour suggère une limitation du tonnage, proposant même que la Royal Navy détruise en échange une partie de sa propre flotte. Cette proposition se voyant refusée, les diplomates anglais la soumettront dans d'autres conférences internationales, notamment en 1925 et à la London Naval Conference de 1930. Un corollaire de ces attitudes est la véritable " euphorie " (le mot est de Hackmann) manifestée par l'Admiralty envers les sonars anglais, les asdics. L'amiral Chatfield sera à plusieurs reprises, en 1918 et en 1920, un des porte-paroles de cet enthousiasme, qualifiant les asdics de «epoch-making achievement » grâce auquel " the hitherto 'undetectable craft' is detectable » et les navires de guerre de la Navy peuvent " keep to the sea as in the golden days $\|^{32}$. En France, les détecteurs de sous-marins ne sont qu'un type d'appareil dans la vaste classe d'objets relevant de l'intercommunication, des échanges par faisceaux ultrasonores horizontaux. Pour d'évidentes raisons géostratégiques, l'importance politique de la lutte contre les sous-marins est bien moindre que pour les Anglais.

L'importance des asdics dans la politique de défense britannique va se traduire par une production en série plus précoce et de plus grande ampleur qu'en France, par plus d'efforts en formation et en recherche, ainsi que par des contraintes de confidentialité bien plus sévères. La sixième flotte de destroyers, soit neuf bâtiment en poste dans l'Atlantique, est entièrement équipée en 1924, et la deuxième le sera à son tour l'année suivante. En 1923 , la fabrication des détecteurs français s'effectue à l'atelier de Toulon, selon une logique davantage artisanale, très loin du mode industriel et standardisé des Britanniques; si l'on exclut les navires destinés à l'étude du matériel, seuls deux bâtiments sont équipés, le Bugalet et le Bapaume, et aucun ne le sera l'année suivante. Au sein de l'ARL, deux des cinq équipes de recherche se consacrent à la détection sous-marine, contre trois ou quatre collaborateurs scientifiques à Toulon. En 1921, la Anti-Submarine School emploie à temps plein sept officiers et trois instructeurs et délivre deux formations différentes. À l'École d'Écoute, créée en 1923, l'enseignement est délivré par le capitaine de frégate et les deux lieutenants de vaisseau composant la CEP-DSM,

32 Cité dans W. Hackmann, Seek and strike, op. cit., p. 127. 
la formation n'étant qu'une de leurs nombreuses tâches. Du côté anglais, les activités des concepteurs et utilisateurs sont régies par le secret-défense. Sur chaque navire, le local abritant les appareils de commande doit être fermé à clef dès que l'asdic n'est pas utilisé, et les parties visibles sur le pont du bateau sont couvertes en cas de visiteurs étrangers. Les sous-marins ont l'interdiction formelle d'utiliser leur asdic à proximité des navires étrangers. Quant aux scientifiques et ingénieurs, ils doivent utiliser le nom de code "asdévite » pour désigner le quartz dans leurs communications écrites ${ }^{33}$.

Le travail du Asdic Group montre à quel point les directives de la Navy ont configuré le développement des détecteurs ultrasonores, tant dans la structure de l'appareil productif que dans l'expérimentation et le prototypage. Dépendant de la Signal School, le centre de TSF de la Navy, ce groupe conduit des recherches amont sur les amplificateurs et sur l'influence de l'eau sur la propagation des ultrasons. Mais il a également pour fonction de contrôler l'atelier de fabrication des quartzs piézoélectriques car la Navy a choisi d'internaliser la production de ce composant hautement stratégique, le reste de l'asdic étant réalisé par un fournisseur et le montage final effectué en interne. Le Asdic Group a bien d'autres tâches liées à la production : contrôler la qualité des quartzs importés, développer des prototypes, préparer des spécifications pour l'industrie, vérifier les pièces construites par les fournisseurs, encadrer l'installation des asdics sur les bâtiments de la flotte et les tests en mer après le montage à bord ${ }^{34}$. Le Asdic Group représente donc une forme de vie scientifique et technique sans équivalent dans le paysage français, car intégrant dans un même régime de pratiques des activités conduites en France dans des espaces différents : les expériences de laboratoire au laboratoire de Toulon, l'usinage et le montage du quartz à la SCAM, les essais en mer et le cadrage de la production à la CEP-DSM. Un autre contrepoint est fourni par la conception des sondeurs à piézoélectricité. Ce travail, dans lequel s'engagent plusieurs physiciens de la Navy, connaît rapidement plusieurs difficultés, toutes liées au caractère confidentiel de cette technologie : l'Admiralty s'oppose à toute prise de brevet et exclut toute commercialisation vers le secteur civil. De surcroît, ces appareils sont concurrencés par les asdics eux-mêmes, dont certains modèles ont une mosaïque orientable vers le bas et peuvent

33 CEP-DSM, Rapports périodiques, 1921-1923, SHM, 1BB8, carton 63; W. Hackmann, Seek and strike, op. cit., pp. 115, 126-129. 34 Ibid., pp. 106-108. effectuer des sondes. Ces conditions génèrent des stratégies de contournement, exploitant des phénomènes différents de la piézoélectricité. En 1925, trois physiciens de l'ARL - C. V. Drysdale, B. S. Smith et E. V. Mackintosh - déposent un brevet basé sur les fréquences audibles et non sur les ultrasons. Commercialisé par Henry Hughes \& Sons, cet appareil ne résiste guère à la concurrence du sondeur Langevin-Florisson quand celui-ci accéde au marché anglais. Un autre effet non couvert par le secret-défense, la magnétostriction des sels de Rochelle, est examiné à partir de 1927 et conduit à un brevet déposé en 1930 par Wood, Smith et J. A. McGeachy ${ }^{35}$. Ce matériau, en revanche, est négligé par les Français; et dans les années 1930, par une ironie de l'histoire, les sondeurs de la SCAM sont sévèrement concurrencés par les appareils Hughes à magnétostriction ${ }^{36}$. Pendant toutes les années vingt, en revanche, les sondeurs français dominent techniquement et commercialement leurs concurrents anglais : la situation est en quelque sorte l'inverse de celle rencontrée pour les asdics.

Pendant l'entre-deux-guerres, la configuration anglaise est marquée par un acteur dominant : bien davantage qu'en France, c'est la Navy qui définit et contrôle les travaux de laboratoire, les stations équipées pour les tests en mer, les formes d'interaction entre les sites, la spécification et la commercialisation du produit, ainsi que les usages stabilisés des objets techniques. Du côté français, la détection des sous-marins ne représente pas un tel enjeu politique et stratégique et, faute de moyen et d'intérêt, la Marine investit peu dans ce type d'équipement. Les sondeurs, en revanche, sont très largement développés, débordent et se répandent dans le secteur civil. Les réseaux socio-techniques sont plus distribués et les acteurs (l'entreprise SCAM, l'École de Physique et Chimie, le laboratoire militaire de Toulon, la CEP-DSM) apparaissent plus autonomes et moins articulés. Chaque entité évoluant selon sa dynamique propre, et les modes de coopération étant moins codifiés et moins robustes, l'ensemble est aussi plus instable. Comme en Angleterre, les équipements

35 A. B. Wood, "Memorial Issue », Journal of the Royal Naval Scientific Service, vol. 20 (1965), 185-284 ; Willem Hackmann, «Sonar research and naval warfare 1914-1954: a case study of twentieth-century science ॥, HSPS, vol.16, partie 1, 1986, pp. 83-110. 36 Les conséquences de la concurrence du sondeur Hughes sont détaillées dans un courrier écrit le 10 octobre 1993 par Bey† (l'administrateur délégué de la SCAM à Paris) et envoyé à Breton (directeur de l'Office national des recherches scientifiques et industrielles et des inventions) pour lui réclamer une diminution des redevances payées aux inventeurs (FL, L141/83). 
permettant les essais en mer représentent une ressource particulièrement décisive et rare. Ils sont pris dans de nombreuses opérations de cadrage qui visent à circonscrire les objets, personnes, pratiques et critères légitimes pour l'évaluation des technologies. La production de ces cadres mobilise de nombreuses paires catégorielles (terrestre/maritime, civil/militaire, théorique/pratique, scientifique/technique ...) dans des arrangements dont on a vu la très grande variété. Une conclusion analogue peut être tirée des monographies réalisées sur l'infrarouge et l'acoustique aérienne. Avec la mobilisation de 1939, les autorités prennent acte de ces difficultés pour rattacher au CNRS le laboratoire qui finit par déménager et devenir le Centre de recherches scientifiques industrielles et maritimes de Marseille. Dès lors, le lien avec les tests opérés sur des navires militaires sera considérablement redéfini ${ }^{37}$.

37 Philippe Narboni, « Recherches appliquées : du Centre d'études de Toulon au Centre de recherches scientifiques de Marseille (1921-1945). Exemple de l'infrarouge et de la détection des avions 1), mémoire de Master 2, EHESS, 2007 ; Claude Gazanhes, « Du laboratoire de la guerre sous-marine de Toulon au laboratoire de mécanique et d'acoustique de Marseille ॥, Revue pour l'histoire du CNRS, n², 2000, pp. 24-35. 